\title{
ESTUDIO DESCRIPTIVO, COMPARATIVO Y CORRELACIONAL DEL ESTADO NUTRICIONAL Y CONDICIÓN CARDIORRESPIRATORIA EN ESTUDIANTES UNIVERSITARIOS DE CHILE
}

\section{DESCRIPTIVE, COMPARATIVE AND CORRELATIONAL STUDY OF NUTRITIONAL AND CARDIO-RESPIRATORY CONDITION OF CHILEAN UNIVERSITARY STUDENTS}

\author{
Hugo Aránguiz A. (1), Vicente García G. (1), Sergio Rojas D. (1), \\ Carlos Salas B. (1), Ricardo Martínez R. (1), Norman Mac Millan K. (2) \\ (1) Departamento de Educación Física, Facultad de Educación. \\ Universidad de Concepción. Concepción, Chile. \\ (2) Escuela de Educación Física. \\ Pontificia Universidad Católica de Valparaíso. Valparaíso, Chile.
}

\begin{abstract}
The aim of this research was to establish the nutritional and cardio-respiratory condition of university students from two important universities of Chile: Pontificia Universidad Católica de Valparaíso (PUCV) and Universidad de Concepción (UdeC). The nutritional and cardio-respiratory condition were evaluated through body mass index (BMI) and the Vo $\mathrm{O}_{2 \max }$ in the 2000 meter walk (UKK) test, respectively. The BMI results of the PUCV showed 78,1\% of students classified as normal $\left(18,5-24,9 \mathrm{~kg} / \mathrm{mt}^{2}\right)$, and the $\mathrm{Vo}_{2 \max }$ showed that $28 \%$ of females were in the regular category (25-30 ml.kg-1.min-1).A34,1\% of males were in the normal category (43-52 ml.kg-1.min-1). In the UdeC the female and male normal level was 79,2\%. The VO2max Index for male and females responders was in the acceptable category of 44,3\% and 40,0\% (31-37 and 34- 42 ml.kg-1.min-1), respectively. The correlation between the nutritional and cardio-respiratory condition established that there is a negative lineal relationship with base $R$-Pearson -0,263 (PUCV) and -0,386 (UdeC), which indicates that as BMI increases the Vo 2max $_{\text {increases. }}$
\end{abstract}

Key words: nutritional condition, cardio-respiratory condition, sedentary.

Este trabajo fue recibido el 9 de Junio de 2009 y aceptado para ser publicado el 30 de Diciembre de 2009.

\section{INTRODUCCIÓN}

Diversos estudios han demostrado que una reducción significativa de la prevalencia de enfermedades infecto contagiosas, se asocia a un paulatino reemplazo por enfermedades crónicas- degenerativas no transmisibles tales como la: hipertensión, hiperlipidemias, diabetes, enfermedades cardiacas y ciertos tipos de cáncer $(1,2)$, lo que podría determinar la mayor cantidad de accidentes cardiovasculares en la población adulta (3). Factores como el sobrepeso, la obesidad y el sedentarismo pueden agravar esta situación (4). En consecuencia, urge la necesidad de analizar la situación presente en un grupo determinado de la población como son los estudiantes universitarios que cursan su primer año de estudios.
En Estados Unidos, dichos estudiantes se encuentran inundados por una cultura popular, de nueva libertad y presión de los pares. Un estudio realizado indica que el 35\% de los estudiantes de educación superior presenta obesidad y un porcentaje de $46 \%$ intenta perder peso, sugiriendo falsas percepciones sobre su imagen corporal. De este porcentaje, sólo uno de cada tres dicen haber recibido en sus lugares de estudio algún tipo de educación relacionado con actividad física y guías de alimentación saludable (5). Además de los trastornos de salud que se acaban de mencionar, no hay que olvidar que las personas obesas sufren, adicionalmente, una marcada discriminación social (6). Por otra parte, el descenso más notorio en la realización de actividad física ocurre en el 
periodo final de la adolescencia y el periodo inicial de la adultez joven (7).

Como consecuencia de una dieta inadecuada y de una actividad física insuficiente, los niveles de obesidad han aumentado en forma alarmante en Chile (8-12). En el año 2003, la Encuesta Calidad de Vida y Salud y en el 2006 la II Encuesta Calidad de Vida y Salud, muestran que la población chilena presenta altos niveles de sobrepeso y obesidad. En la primera encuesta $38 \%$ tiene sobrepeso, $22 \%$ es obeso y $1,3 \%$ presenta obesidad mórbida, lo que en total suma un $61,3 \%$. En la encuesta efectuada en el año 2006 el $40 \%$ se percibe con sobrepeso y un $7,4 \%$ como obesa (9). Estas cifras son similares a países desarrollados. En efecto, en el caso de Estados Unidos un $64 \%$ del total de la población presentaba el año 2000 un estado nutricional calificado como deficiente (13). Estas cifras son indicadores respecto al estado nutricional de la población chilena, la que no se encuentra en gran proporción en los rangos de normalidad según tablas internacionales $(14,15)$.

A finales de la década de los ' 60 y principios de los '70 emerge un concepto de condición física más próximo al ámbito de la salud, lo que propicia una cierta ruptura ideológica al tomar como propósito principal el bienestar del individuo por encima del objetivo tradicional basado en el rendimiento deportivo. Un estudio realizado en año 1995, utiliza el concepto de condición cardiorrespiratoria como método para el estudio del estado de salud de las personas. Para ello utilizó el consumo de oxígeno y la frecuencia cardíaca como algunas de las principales variables para el estudio de dicha relación (16).

La expresión $\mathrm{Vo}_{2 \text { máx. }}$ (ml-1.kg-.min-1) es un indicador de capacidad aeróbica, cuyo valor numérico se relaciona con un nivel físico de la eficiencia de utilización de la energía aeróbica (17). Su valor absoluto depende de la capacidad física de cada individuo, es un parámetro fisiológico que expresa la cantidad de oxígeno que consume o utiliza el organismo (18).

La actividad física pasa a ser considerada como un componente importante dentro de un estilo de vida saludable, ya que la ejecución de actividades físicas sistemáticas y de cierta intensidad constituye un factor de protección y prevención de diferentes trastornos de la salud, por los importantes beneficios fisiológicos, psicológicos y sociales asociados a la actividad física (19-22). Estudios recientes muestran que tener una buena condición física reduce la probabilidad de padecer enfermedades como el cáncer y la diabetes tipo 2 relacionadas a personas sedentarias obesas $(23,24)$.

En diversas investigaciones internacionales se ha demostrado que una vez comenzados los estudios de educación superior disminuye la intensidad y frecuencia de actividad física que desarrollan las personas, especialmente los adultos jóvenes (25). Este antecedente puede servir para que las autoridades del área de la educación superior reflexionen y consideren medidas que permitan entregar una educación integral, considerando el desarrollo de actividades deportivas o físicas durante los años de estudios superiores como se hace en la actualidad en Japón (26) donde la realización de actividad física durante los años de estudios es obligatoria y un requisito para su culminación.

El propósito de esta investigación fue determinar la incidencia del nivel de condición cardiorrespiratoria como capacidad funcional medida por su consumo máximo de oxígeno $\left(\mathrm{V}_{\mathrm{O}_{\text {máx }}}\right)$. El estado nutricional determinado a través del Indice de Masa Corporal (IMC) de estudiantes de la Pontificia Universidad Católica de Valparaíso y de la Universidad de Concepción.

\section{SUJETOS Y MÉTODOS}

En ambas universidades la muestra de la investigación fue de tipo probabilística, representativa, estratificada y proporcional para ambos géneros. En la Pontificia Universidad Católica Valparaíso se evaluaron 64 alumnos de los cuales 20 eran mujeres y 44 varones. En la Universidad de Concepción se evaluaron 245 alumnos que corresponden a 115 mujeres y varones.

Para medir el peso y la estatura se utilizó la balanza marca SECA, modelo 713, con escala de 5 a $200 \mathrm{Kg}$. y precisión de $0,1 \mathrm{Kg}$. y el estadiometro marca HOLTAIN. Se determinó el IMC; este indicador es utilizado para valorar el estado nutricional. El procedimiento consiste en medir el peso corporal en kilogramos dividido por la estatura en metros elevado al cuadrado de cada individuo. De acuerdo a tablas aceptadas por la Organización Mundial de la Salud (OMS) se presentan cinco categorías (14): bajo peso, peso normal, sobrepeso, obesidad, obesidad mórbida (14).

Se utilizó el test de caminata (2.000 metros) UKK para evaluar la condición cardiorrespiratoria $\left(\mathrm{VO}_{2 \text { máx }}\right)$. El test se aplicó en una pista atlética de 400 metros de perímetro. Cada alumno debió dar cinco vueltas a la pista de manera vigorosa y enérgica. Los datos que se utilizaron para establecer la predicción del consumo máximo de oxígeno corresponden a las siguientes variables: índice de masa corporal, edad, tiempo y frecuencia cardíaca en realizar la prueba. Los estándares internacionales para realizar la clasificación fueron: baja, regular, aceptable, buena y excelente (15). Para la medición de tiempo y frecuencia cardiaca se utilizo el pulsímetro marca POLAR modelo FS1.

Con los datos obtenidos se realizó un análisis estadístico, utilizando la correlación de Pearson entre las va- 
riables (IMC y $\mathrm{Vo}_{2 \text { máx }}$ ) de nivel de medición intercalar y la T de Student para establecer el grado de significación. Además, se relacionó de acuerdo a porcentajes, medias, desviación estándar, de ambas universidades.

Para el análisis estadístico de los datos se utilizaron los siguientes softwares estadísticos: SPSS 15, MINITAB 14, R2.6 y Microsoft Excel.

\section{RESULTADOS}

En la tabla 1, se presentan los resultados de estadísticos descriptivos de las variables utilizadas, en el género femenino los resultados muestran homogeneidad. Pero en el género masculino se detectaron diferencias en sus medias, en la PUCV existe una superioridad de 5,43 ml.kg-1.min-1 en el $V_{o_{2} \text { máx. }}$ y de 2,26 puntos menos en el IMC que los alumnos de la UdeC.

En la tabla 2 figuran el estado nutricional de la muestra que, se han clasificado de acuerdo a los criterios de la OMS (16), que sobre del 75\% de las muestras evaluadas se encuentran con un IMC clasificado como normal, y $1,6 \%$ presenta obesidad en la PUCV, y un mayor porción mayor tiene la UdeC con un 5,3\% de obesidad.

TABLA 1

Distribución de los alumnos evaluados, por universidad y género

\begin{tabular}{|c|c|c|c|}
\hline \multirow{9}{*}{$\begin{array}{l}\text { Pontificia } \\
\text { Universidad } \\
\text { Católica } \\
\text { de } \\
\text { Valparaíso }\end{array}$} & & $\begin{array}{c}\text { FEMENINO }(n=20) \\
\text { MEDIA } \pm \text { D.E. }\end{array}$ & $\begin{array}{c}\text { MASCULINO }(n=44) \\
\text { MEDIA } \pm \text { D.E. }\end{array}$ \\
\hline & Peso $(\mathrm{Kg})$ & $9,50 \pm 5,83$ & $73,59 \pm 12,11$ \\
\hline & Estatura (Mt) & $1,63 \pm 0,08$ & $1,75 \pm 0,60$ \\
\hline & IMC $\left(\mathrm{kg} / \mathrm{mt}^{2}\right)$ & $22,62 \pm 2,73$ & $20,95 \pm 3,04$ \\
\hline & Edad (Años) & $19,85 \pm 2,32$ & $19,67 \pm 1,10$ \\
\hline & Tiempo (Seg.) & $19,22 \pm 2,26$ & $17,97 \pm 1,76$ \\
\hline & Frecuencia Cardiaca (pulsac./min) & $152,90 \pm 24,19$ & $143,34 \pm 25,44$ \\
\hline & $\mathrm{VO}_{2 \text { máx }}(\mathrm{ml} . \mathrm{kg}-1 . \mathrm{min}-1)$ & $30,52 \pm 7,06$ & $42,84 \pm 10,05$ \\
\hline & & $\begin{array}{c}\text { FEMENINO }(\mathbf{n}=\mathbf{1 5 5}) \\
\text { MEDIA } \pm \text { D.E. }\end{array}$ & $\begin{array}{l}\text { MASCULINO }(n=130) \\
\text { MEDIA } \pm \text { D.E. }\end{array}$ \\
\hline \multirow{7}{*}{$\begin{array}{l}\text { Universidad } \\
\text { de } \\
\text { Concepción }\end{array}$} & Peso $(\mathrm{Kg})$ & $57,20 \pm 6,86$ & $69,10 \pm 9,60$ \\
\hline & Estatura (Mt) & $1,59 \pm 0,06$ & $1,73 \pm 0,70$ \\
\hline & IMC $\left(\mathrm{kg} / \mathrm{mt}^{2}\right)$ & $22,40 \pm 2,78$ & $23,21 \pm 3,08$ \\
\hline & Edad (Años) & $18,85 \pm 0,85$ & $19,10 \pm 1,11$ \\
\hline & Tiempo (Seg.) & $19,22 \pm 1,37$ & $18,08 \pm 1,46$ \\
\hline & Frecuencia Cardiaca (pulsac./min) & $155,73 \pm 15,62$ & $154,82 \pm 20,93$ \\
\hline & $\mathrm{VO}_{2 \text { máx }}(\mathrm{ml} . \mathrm{kg}-1 . \mathrm{min}-1)$ & $30,41 \pm 4,37$ & $37,41 \pm 9,18$ \\
\hline
\end{tabular}

\section{TABLA 2}

Porcentajes del estado nutricional de los alumnos de las universidades, según su variable y género.

\begin{tabular}{|c|c|c|c|c|c|c|c|}
\hline & \multirow[b]{2}{*}{ CATEGORIAS } & \multicolumn{3}{|c|}{ PUCV $(n=64)$} & \multicolumn{3}{|c|}{ UdeC $(n=245)$} \\
\hline & & Femenino & Masculino & $\begin{array}{c}\text { Total } \\
\text { Ponderado }\end{array}$ & Femenino & Masculino & $\begin{array}{c}\text { Total } \\
\text { Ponderado }\end{array}$ \\
\hline IMC & Bajo $(18,5)$ & $0 \%$ & $13,6 \%$ & $9,4 \%$ & $3,5 \%$ & $1,5 \%$ & $2,4 \%$ \\
\hline \multirow[t]{3}{*}{$\mathrm{Kg} / \mathrm{mt}^{2}$} & Normal $(18,5-24,9)$ & $80,0 \%$ & $77,3 \%$ & $78,1 \%$ & $80,0 \%$ & $78,5 \%$ & $79,2 \%$ \\
\hline & Sobrepeso $(25-29,9)$ & $20,0 \%$ & $6,8 \%$ & $10,9 \%$ & $13,9 \%$ & $12,3 \%$ & $13,1 \%$ \\
\hline & Obesidad $(30-39,9)$ & $0 \%$ & $2,3 \%$ & $1,6 \%$ & $2,6 \%$ & $7,7 \%$ & $5,3 \%$ \\
\hline
\end{tabular}


Los varones presentan en la UdeC $18,4 \%$ sobrepeso y obesidad, y en la PUCV obtiene un $12,5 \%$.

En la tabla 3 presenta las proporciones de consumo máximo de oxigeno, la PUCV exhibe una mayor proporción en las categorías aceptable $(25,0 \%)$ y buena $(28,1 \%)$, en la UdeC por su parte presenta en las categorías aceptable $(42,0 \%)$ con un $17 \%$ más que los alumnos de la PUCV, y regular $(26,5 \%)$, también se observó $10,9 \%$ de los alumnos de PUCV en la categoría baja, mientras que la UdeC presenta un $14,3 \%$ en la misma categoría.
En el gráfico 1, se observa el IMC en la PUCV ostenta una mayor curtosis, alcanzando una mayor densidad, por su parte las alumnas de la UdeC presentan una densidad más baja con valores más cercanos a la clasificación normal. En el grafico 2 la variable $\mathrm{Vo}_{2 \text { máx. }}$ la PUCV tiene una densidad media (mesocúrtica), reflejada en la dispersión de la curva y la concentración con rangos dispersos de 20 a 30 ml.kg-1.min-1, por su parte las alumnas de la UdeC presenta una mayor densidad (leptocúrtica), agrupándose en los rangos de 28 a 37 ml.kg-1.min-1 más cercano a la clasificación aceptable.

\section{TABLA 3}

Porcentaje del consumo máximo de oxígeno de los alumnos de las universidades, según su variable y género.

\begin{tabular}{|c|c|c|c|c|c|c|c|c|c|c|}
\hline \multirow{2}{*}{$\begin{array}{l}\mathrm{Vo}_{2 \text { máx. }} \\
\text { ml.kg-1.min-1 }\end{array}$} & \multicolumn{2}{|c|}{ Baja } & \multicolumn{2}{|c|}{ Regular } & \multicolumn{2}{|c|}{ Aceptable } & \multicolumn{2}{|c|}{ Buena } & \multicolumn{2}{|c|}{ Excelente } \\
\hline & $<24)$ & $<25$ & 25- 30 & 26- 33 & 31- 37 & 34- 42 & 38- 48 & 43- 52 & $>48$ & $>52$ \\
\hline PUCV Femenino & $25,0 \%$ & & $35,0 \%$ & & $25,0 \%$ & & $15,0 \%$ & & $0,0 \%$ & \\
\hline UdeC Femenino & $14,8 \%$ & & $37,4 \%$ & & $44,3 \%$ & & $3,5 \%$ & & $0,0 \%$ & \\
\hline PUCV Masculino & & $4,5 \%$ & & $15,9 \%$ & & $25,0 \%$ & & $34,1 \%$ & & $20,5 \%$ \\
\hline UdeC Masculino & & $13,8 \%$ & & $16,9 \%$ & & $40,0 \%$ & & $24,6 \%$ & & $4,6 \%$ \\
\hline PUCV Total & 10 & & & & & & & & & $1 \%$ \\
\hline UdeC Total & 14 & & & & & & & & & $4 \%$ \\
\hline
\end{tabular}

\section{GRÁFICO 1}

Densidades de índice de masa corporal en el grupo femenino.

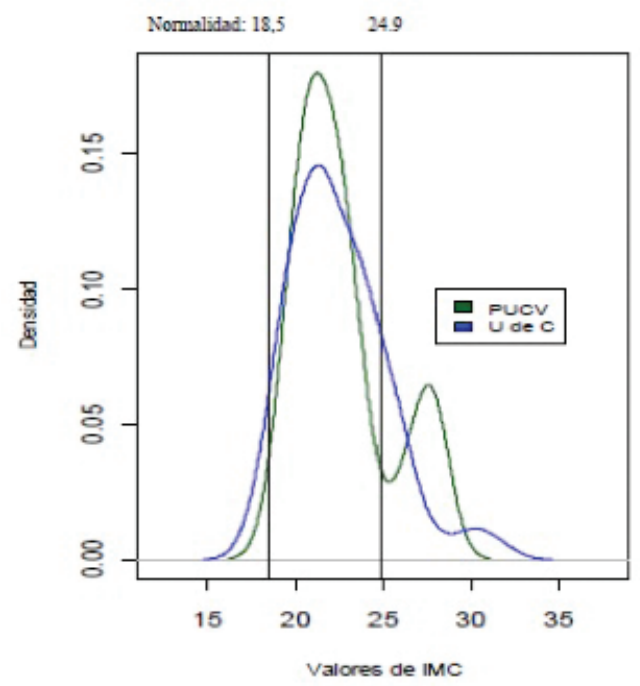

\section{GRÁFICO 2}

Densidades de consumo de oxígeno en el grupo femenino.

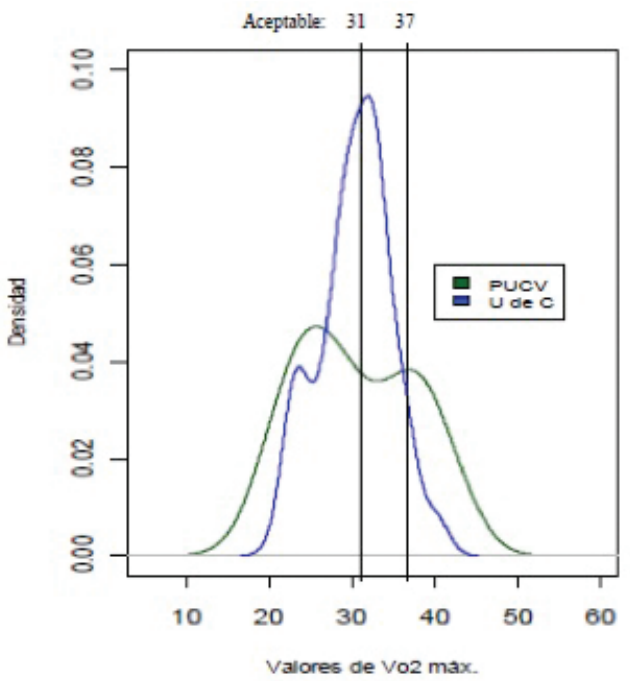


En el gráfico 3 se aprecia en IMC de varones, la PUCV presenta una densidad más cercana a la clasificación Normal que los alumnos de la UdeC, el gráfico 4 el $V_{o_{2 m a ́ x}}$ se observó que la PUCV esta inclinada hacia la derecha lo que indica que sus estudiantes presentan clasificaciones superior a la clasificación normal, y la UdeC se observa más cercana a la clasificación aceptable, ambas universidades la curva es mesocúrtica.

En el gráfico 5, se observa la dispersión de las variables IMC y $\mathrm{Vo}_{2}$ máx. de los estudiantes universitarios, los alumnos de la PUCV se exhiben más dispersos, respecto a los alumnos de la UdeC.

Los resultados de la correlación de Pearson entre las universidades son negativas, mediana y de baja intensidad.

Los datos obtenidos de la PUCV no presentan significancia, presentándose negativa y débil, y en la UdeC la correlación se exhibe negativa y de mediana intensidad.

\section{DISCUSIÓN}

El estudio realizado tuvo como objetivo establecer las relaciones respecto a las variables de estado nutricional (IMC) y consumo máximo de oxígeno (Vo2máx.), que inciden tanto en la salud física como psicológica, incrementando así la calidad de vida. Es importante

\section{GRÁFICO 3}

\section{Densidades de índice de masa corporal en el grupo masculino.}

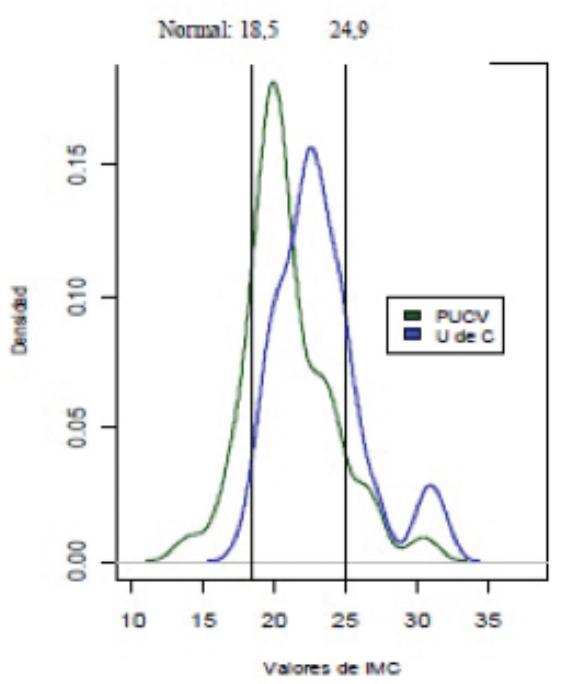

mencionar que estas variables están relacionadas con la alimentación y la práctica de actividad física regular que favorece e instaura estilos de vida más saludables y reduce o elimina los factores de riesgo asociados al sedentarismo $(23,24)$.

Los resultados encontrados respecto al estado nutricional en estudiantes universitarios PUCV y UdeC, fueron $78,1 \%$ y $79,2 \%$ con un IMC en la categoría de normal, $10,9 \%$ y $13,1 \%$ con sobrepeso y $2,3 \%$ y $5,3 \%$ obesidad, respectivamente. Estos antecedentes son similares a un estudio realizado en Colombia (2008) con estudiantes universitarios que presentaban un $80,0 \%$ de clasificación normal y un sobrepeso $10,1 \%$ y $1 \%$ de obesidad27, y que coincide con una investigación de similares características de UNAM (2006), con un 58,9\% clasificados en la categoría normal y un $19,7 \%$ de sobrepeso $17,6 \%$ y obesidad 2,1\%28. Aunque la obesidad mostró una prevalencia muy baja en este estudio no se debe olvidar que este trastorno está asociado a factores de riesgo como hipertensión, lislipidemias, sedentarismo y diabetes.

En un estudio realizado en España (29), los valores medios encontrados de VO2máx. para adolescentes de género femenino son de $43,0 \pm 5,16 \mathrm{ml} / \mathrm{kg}-1 / \mathrm{min}-1$, $\mathrm{y}$ para los adolescentes del género masculino son de 47,5 $\pm 5,29 \mathrm{ml} / \mathrm{kg}-1 / \mathrm{min}-1$, que se sitúan en el umbral de

\section{GRÁFICO 4}

\section{Densidades de consumo de oxígeno en el grupo masculino.}

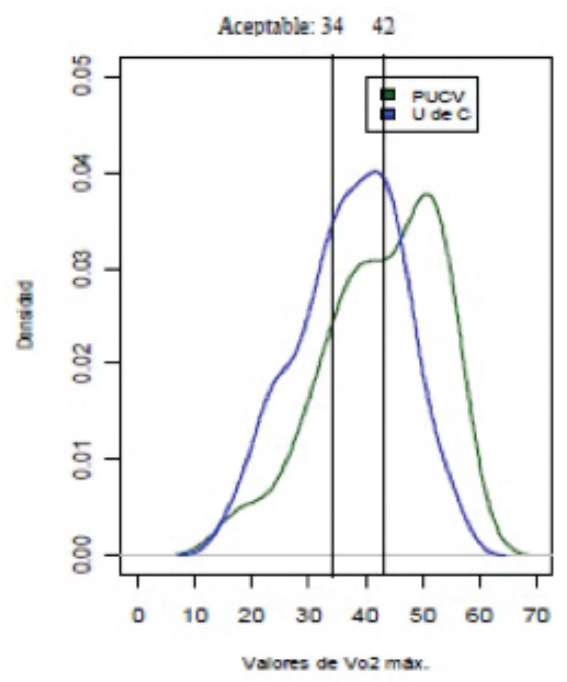


salud aeróbica de 35 y $42 \mathrm{ml} / \mathrm{kg}-1 / \mathrm{min}-1$ considerado por el Cooper Institute for aerobics research30. En nuestro estudio los valores del $\mathrm{Vo}_{2 \text { máx. }}$ para el género femenino y masculino las medias fueron $30,42 \pm 5,7$ y 40,12 \pm 9,6 ml.kg-1.min-1 respectivamente, que son significativamente menores al estudio anteriormente mencionado.

La correlación existente entre las variables, estado nutricional y condición cardiorrespiratoria la PUCV presenta un R -0,26 y la UdeC R -0,39. Estos datos reflejan que es baja y negativa. Al analizar los datos del género femenino se observan las siguientes dife- rencias: la PUCV -0,055 y UdeC -0,504, y el grupo masculino presenta resultados similares al femenino: la PUCV $-0,182$ y la UdeC $-0,522$. Se deduce que en la universidad de la quinta región no existe asociación lineal entre las variables, mientras que la Universidad de Concepción tiene una relación media intensidad y negativa. Esto quiere decir que los alumnos con índices nutricionales mayores a la clasificación normal, (sobrepeso y obesidad), presentan una capacidad cardiorrespiratoria inferior (Baja, Regular). El estudio realizado por Ross y Katzmaryk (31), quienes en una muestra de 7.537 varones y mujeres refieren que los

\section{GRÁFICO 5}

\section{Diagrama de dispersión entre el índice de masa corporal y consumo máximo} de oxígeno de las universidades.

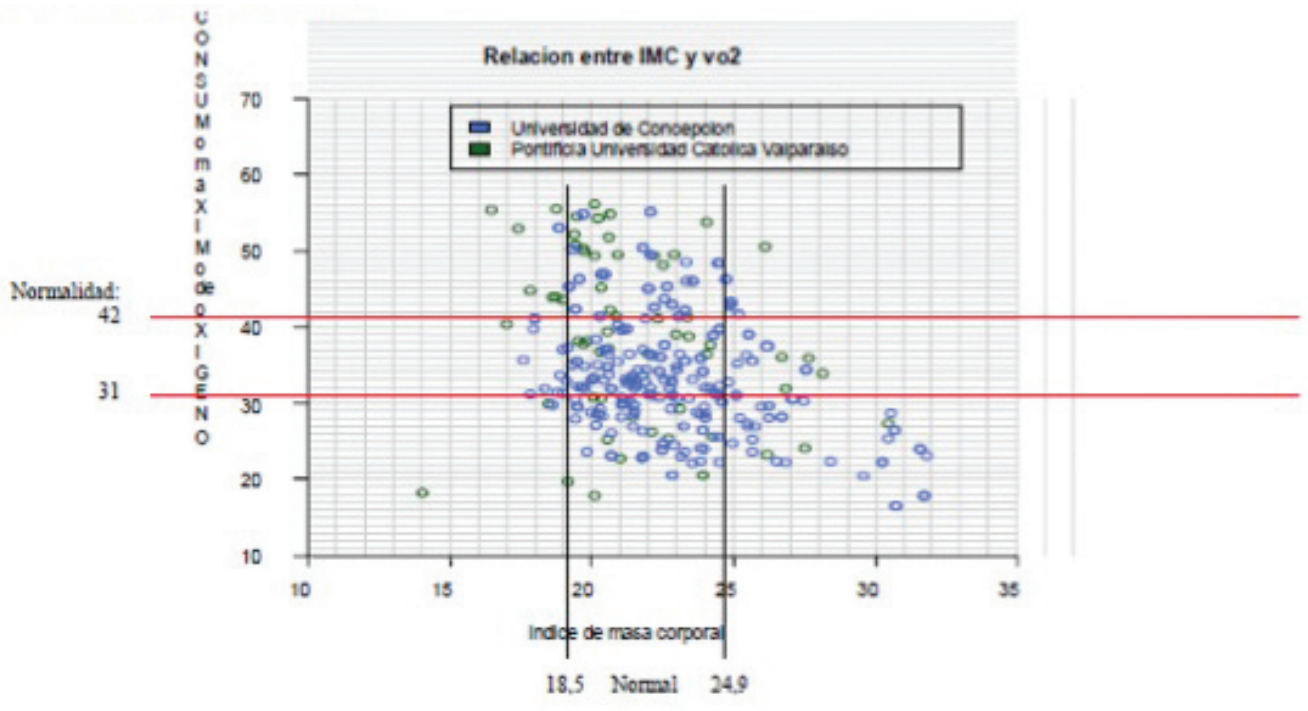

\section{TABLA 4}

Correlación entre IMC y Vo2máx., en ambas universidades, y separadas por género.

\section{Universidad}

Coeficiente

$\mathrm{R}$ de Pearson

Estrato

$\mathrm{R}$ de Pearson

\section{PUCV}

Total muestra

$-0,26(*)$
Género

femenino

$-0,055$
Género

masculino

$-0,182$
UdeC

Total muestra $-0,39$ (*)

Nivel de significación: $5 \%$

(*): Significativo. 
sujetos con mayor índice cardiorrespiratoria tenían menor cantidad de masa grasa total y abdominal, concuerda con los resultados encontrados en la presente investigación.

Estos antecedentes sugieren que el grado de condición física podría atenuar los factores de riesgo relacionados con el sobrepeso y la obesidad, destacando la importancia de la actividad física regular en la prevención y el tratamiento del sobre- peso y las enfermedades cardiovasculares (32).

Es necesario tener en cuenta que los jóvenes universitarios se encuentran en un periodo crítico en sus vidas, ya que tienden a disminuir su nivel de actividad física, aumentar su peso y por lo mismo, tienden a la práctica de conductas para controlar el peso, como utilizar dietas inadecuadas, gulas y ayunos que favorecen el riesgo tanto a desórdenes alimentarios como riesgo para obesidad y sobrepeso (33-35). Al respecto, se tiene información adecuada sobre patrones y conductas de alimentación, el hábito de actividad física, la vigilancia del peso y la detección de trastornos de la alimentación, ayudaría al estudiante a elegir opciones que le favorezcan tener un estilo de vida saludable.

La investigación tuvo como propósito establecer un conocimiento objetivo, sobre la importancia que tiene la condición cardiorrespiratoria como indicador de condición física y su relación con el estado nutricional de estudiantes universitarios, encontrándose una relación significativa que indica que una condición cardiorrespitoria, ubicada en el umbral de salud aeróbica30 es asociada a un IMC en la categoría normal, lo que corrobora antecedentes de estudios realizados respecto a la correlación entre $\mathrm{Vo}_{2 \max }$ e IMC. Este estudio entrega antecedentes de jóvenes universitarios respecto a las variables en estudio para proponer estrategias de práctica de forma regular y sistemática de actividad física como componente importante de factor protector de la salud relacionada con la mantención de la condición física saludable.

\section{RESUMEN}

El siguiente estudio descriptivo correlacional fue realizado con el objeto de determinar el estado nutricional y el nivel cardiorrespiratorio de los alumnos de las universidades Pontificia Universidad Católica de Valparaíso (PUCV) y la Universidad de Concepción (UdeC). El estado nutricional se evaluó por el Indice de Masa Corporal (IMC) y la condición cardiorrespiratoria por el consumo máximo de oxígeno $\left(\mathrm{Vo}_{\text {2máx }}\right)$, mediante el test de la caminata de 2.000 metros (UKK). En la PUCV, los resultados del IMC presentan 78,1\% clasificado como normal $\left(18,5-24,9 \mathrm{~kg} / \mathrm{mt}^{2}\right)$, mientras que el
$28 \%$ de las mujeres mostró una mayor frecuencia en la clasificación de regular en el Vo2máx. (25- 30 ml.kg-1. min-1). El 34,1\% de los varones se encontró en la categoría normal (43- 52 ml.kg-1.min-1). Por su parte, en la UEDC, el IMC, tanto de mujeres como de varones se ubicaron en la categoría normal $(79,2 \%)$. El Vo 2máx. de mujeres como varones presentó su mayor concentración en la categoría aceptable (44,3\% y 40,0\%) (31- 37 y 34- 42 ml.kg-1.min-1) respectivamente. La correlación entre estado nutricional y condición cardiorrespiratoria estableció una relación lineal negativa en base $\mathrm{R}$ de Pearson -0,263 (PUCV) y -0,386 (UdeC), que significa que a mayor IMC menor $\mathrm{Vo}_{2 \text { máx. }}$.

Palabras clave: Estado nutricional, condición cardiorrespiratoria, sedentarismo.

Dirigir la correspondencia a:

Dr. Hugo Aránguiz A.

Departamento de Educación Física

Universidad de Concepción

Casilla $160-\mathrm{C}$

Correo 3

Fono / fax: (041) 2257226

E- mail: harangui@udec.cl

Agradecimientos: Investigación financiada por Proyecto Semilla No 205163010- 1S Universidad de Concepción. Pontifica Universidad Católica de Valparaíso. Chile.

\section{BIBLIOGRAFÍA}

1. Popkin BM. An overview on the nutrition transition and its health implications: the Bellagio meeting. Public Health Nutr 2002; 5: 93-103.

2. Popkin BM. The shift in stages of the nutrition transition in the developing world differs from past experiences Public Health Nutr 2002b; 5: 205- 214.

3. Landrove O, Gámez AI. Transición epidemiológica y las enfermedades crónicas no transmisibles en las Américas y en Cuba: el programa de intervención cubano. Reporte Técnico de Vigilancia 2005; 10 (6): 1-18.

4. Field A, Coakley E, Must A, Spadano J, Laird N, Dietz $\mathrm{W}$ and cols. Impact of overweight on the risk of developing common chronic diseases during a 10-year period. Arch Intern Med 2001; 161:158186.

5. Lowry R., Galuska D. A., Fulton J. E., Wechsler H., Kann L., and Collins J. L. Physical activity, food choice, and weight management goals and practices among U.S. college students. Am J Preventive Med 
2000; 18(1): 18-27.

6. Anderson, D.A. y Wadden, T.A. Treating the obese patient: Suggestions for primary care practice. Arch Family Med 1999; 8: 156-167.

7. Dishman, R. K. Mental Health. En V. Seefeldt (ed.), Physical activity and well-being. Reston: American Alliance of Health, Physical Education, Recreation and Dance; 1985

8. Ministerio de Salud. Instituto Nacional de Estadísticas. Encuesta Nacional de Salud. Chile. 2003

9. Ministerio de Salud. Departamento de Epidemiología. II Encuesta de calidad de vida y salud. Chile. 2006.

10. Chiledeportes. Instituto Nacional del Deporte y el Observatorio Social de la Universidad Alberto Hurtado. Encuesta nacional de hábitos de actividad física y deporte en la población igual o mayor a 18 años. Chile. 2006.

11. Instituto Nacional de Estadísticas. Encuesta de Caracterización Socio Económica Nacional Chile. 2000.

12. Vio F., Albala C. Nutrition transition in Chile: A case study. In: Globalization of food systems in developing countries: impact on food security and nutrition. Food and Agriculture Organization of the United Nations, Rome. FAO. Food Nutr Paper 2004; 83: 275-284.

13. Flegal, K. M. Margaret D. Carroll, MS; Cynthia L. Ogden, PhD; Clifford L. Johnson, MSPH. Prevalence and trends in obesity among US adults (1999- 2000) 2002; 288:1723-1727.

14. World Health Organization. The use and interpretation of Anthropometry. WHO. Technical report series. N. 854. Geneva, 1999.

15. American Heart Association. Exercise testing and training of apparently healthy individuals: a handbook for physicians. Nueva York: 1972; pp 15.

16. Shephard R.J. Physical activity, fitness and health: the current consensus. Quest. 1995; 47: 288-303.

17. Arstrand P-O, Rodahl K. Fisiología del trabajo físico. Bases fisiológicas del ejercicio. $3^{\mathrm{a}}$. Ed. Buenos Aires: Argentina, Medica Panamericana, 1996, pp. 311.

18. Bernard R Ch. Excercise stress testing. Branwald E Heart disease. 5ta ed. USA: Saunders; 1997.

19. Kulinna, P., \& Zhu, W. Fitness portfolio calibration for first- through sixth- grade children. Res Quarterly Exercise Sport 2001; 72 (4), 324- 334.

20. Demarco, T.; Sidney, K. Enhancing children's participation in physical activity. J School Health 1989; 59, (8): 337- 340.

21. Sánchez- Barrera, M.; y cols. Patrones de actividad física de una muestra española. Rev Psicol Deporte 1995; n. 7- 8: 51-71.

22. Segura, J. Hábitos de actividad física en estudiantes universitarios. Psicología de la Actividad Física y el Deporte, Tomo I. Murcia 1999.

23. Evenson, K., Steven J., Cai J., Thomas R. The effect on Cardiorespiratory Fitness and Obesity on Cancer Mortality in Women and Men. Med Science Sports Exercise; 2003 Vol. 35 (2): 270-277.

24. Lee D., Sui X., Church T., Lee I*Min y Blair S. Associations of Cardiorespiratory Fitness and Obesity With Risks of Impaired Fasting Glucose and Type 2 Diabetes in Men. Diabetes Care; 2009; 32 (2): 257-262.

25. Timiras P.S. Bases fisiológicas del envejecimiento y geriatría. Edit. Masson. Barcelona: 1997.

26. Tazuke Shunichi. The mood before and after physical activity in two different physical education classes at university in Japan. Doshisha University, Japan. (Jyväskylä, Finland). 12th Annual Congress of the ECSS. 11-14 July 2007.

27. Melier Vargas- Zárate, Fabiola Becerra- Bulla y Edgar Prieto- Suárez. Evaluación Antropométrica de Estudiantes Universitarios en Bogotá. Rev Salud Pública, 2008; 10 (3): 433-442.

28. Morán I., Cruz V., Iñárritu M. El índice de masa corporal y la imagen corporal percibida como indicadores del estado nutricional en universitarios. Universidad Autónoma de México. Departamento de Salud Pública, Facultad de Medicina. 2007; 50 (2) 125- 151.

29. Casajus J., Leiva M., Ferrando J., Moreno L., Aragones M., Ara I. Relación entre la condición física cardiovascular y distribución de grasa en niños y adolescentes. Apunts. Medicina L'Esport. 2006; 149: 7- 14.

30. The Cooper Institute for Aerobics Research, FITNESSGRAM test administration manual. Champaign: Human Kinetics; 1999; pp 38- 39.

31. Ross R., Katzmarzyk PT. Cardiorespiratory fitness is associated with diminished total and abdominal obesity independent of body mass index. Inter $\mathbf{J}$ Obes 2003; 27:204-10.

32. United States Department of Health and Human Services. Physical activity and health: a report of the Surgeon General. Atlanta, Georgia: Department of Health and Human Services, Centers for Disease Control and Prevention, National Center for Chronic Disease Prevention and Health Promotion, The Presidents' Council on Physical Fitness and Sports; 1996.

33. Nemet, D., Barkan, S., Epstein, Y., et al. Short and 
long term beneficial effects of a combined dietaryBehavioral-physical activity interventions for the treatment of childhood obesity. Pediatrics 2005; 115: 443- 449.

34. Nothwehr, F., Attitudes and behaviors related to weight control in two diverse populations. Preventive Medicine 2004; 39: 674-680.
35. Field A., Cheung, L., Wolf, A., et al. Exposure to the Mass Media and Weight Concerns Among Girls. Pediatrics 1999; 103: 1-5.

36. Chiang- Salgado M, Casanueva V, Cid-Cea X, González U, Olate P, Níkel F, Revello L. Factores de riesgo cardiovascular en estudiantes universitarios chilenos. Sal Pub Mex 1999; 41(6):444-451. 